\title{
High Performance H-mode Plasmas at Densities Above the Greenwald Limit
}

M.A. Mahdavi, ${ }^{1}$ T.H. Osborne, ${ }^{1}$ A.W. Leonard, ${ }^{1}$ M.S. Chu, ${ }^{1}$, E.J. Doyle, ${ }^{2}$ M.E. Fenstermacher, ${ }^{3}$ G.R. McKee, ${ }^{4}$ G.M. Staebler, ${ }^{1}$ T.W. Petrie, ${ }^{1}$ M.R. Wade, ${ }^{5}$ S.L. Allen, ${ }^{3}$ J.A. Boedo, ${ }^{6}$ N.H. Brooks, ${ }^{1}$ R.J. Colchin, ${ }^{5}$ T.E. Evans, ${ }^{1}$ C.M. Greenfield, ${ }^{1}$ G.D. Porter, ${ }^{3}$ R.C. Isler, ${ }^{5}$ R.J. La Haye, ${ }^{1}$ C.J. Lasnier, ${ }^{3}$ R. Maingi, ${ }^{5}$ R.A. Moyer, ${ }^{6}$ M.J. Schaffer, ${ }^{1}$ P.G. Stangeby, ${ }^{7}$ J.G. Watkins, ${ }^{8}$ W.P.West, ${ }^{1}$ D.G. Whyte, ${ }^{6}$ N.S. Wolf ${ }^{3}$

${ }^{1}$ General Atomics, P.O. Box 85608, San Diego, California 92186-5608, USA email: mahdavi@fusion.gat.com

2University of California, Los Angeles, California, USA

${ }^{3}$ Lawrence Livermore National Laboratory, Livermore, California 94550, USA

${ }^{4}$ University of Wisconsin, Madison, Wisconsin, USA

5Oak Ridge National Laboratory, Oak Ridge, Tennessee 37381, USA

6 University of California, San Diego, California, USA

${ }^{7}$ University of Toronto Institute for Aerospace Studies, Toronto, Canada

${ }^{8}$ Sandia National Laboratories, P.O. Box 5800, Albuquerque, New Mexico, USA

\begin{abstract}
Densities up to 40 percent above the Greenwald limit are reproducibly achieved in high confinement $\left(\mathrm{H}_{\text {ITER89p }}=2\right)$ ELMing H-mode discharges. Simultaneous gas fueling and divertor pumping were used to obtain these results. Confinement of these discharges, similar to moderate density H-mode, is characterized by a stiff temperature profile, and therefore sensitive to the density profile. A particle transport model is presented that explains the roles of divertor pumping and geometry for access to high densities. Energy loss per ELM at high density is a factor of five lower than predictions of an earlier scaling, based on data from lower density discharges.
\end{abstract}

\section{Introduction}

A critical fusion reactor issue is the density limit and density effects on plasma confinement and stability. Both the ignition criteria and effective radiative heat dispersal require operating at sufficiently high densities. However, the well known but poorly understood Greenwald density limit scaling law [1,2] indicates that in reactor grade devices access to the desired operating densities would be problematic. Another area of concern is observations made on several tokamaks, notably JET and JT60-U [3], that confinement in H-mode plasmas degrades at higher densities well before reaching the Greenwald limit $\left(\mathrm{n}_{\mathrm{GW}} \equiv \mathrm{I}_{\mathrm{p}}(\mathrm{MA}) /\right.$ $\pi \mathrm{a}^{2}\left(10^{20} \mathrm{~m}^{-3}\right)$. In this paper we will present the results and interpretation of experiments on the DIII-D tokamak [4] where densities up to $1.4 \mathrm{x} \mathrm{n}_{\mathrm{GW}}$ were reproducibly obtained in H-mode plasmas without confinement degradation $\left(\mathrm{H}_{\text {ITER }} 89 \mathrm{p}=2\right)$. A combination of gas fueling and divertor pumping is used to access these densities. A highly desirable feature of these discharges is that the amplitude of Type-I ELMs is a factor of 5 lower than that indicated by a recent multi-device study at lower densities [5]. These results show that there is no fundamental limitation preventing high confinement $\mathrm{H}$-mode operation above the Greenwald limit, even with gas fueling.

The remainder of this paper is organized in four sections. The experimental arrangement and main experimental observations are presented in Section 2. The confinement and stability of the high density discharges is discussed in Section 3. In this section it is shown that confinement behavior is consistent with a stiff transport model, and that the time dependence of the stored energy is mainly a consequence of the time evolution of the density profile. The highest densities achieved were limited by the onset of a $3 / 2$ tearing mode, discussed in Section 3.B. In Section 4 we discuss access to the high density regimes. A simple particle transport model is developed to help understand the roles of divertor pumping and the distribution of gas fueling source in access to high densities. 


\section{Observations}

The experiments presented here were carried out in the single-null configuration at low triangularity, $\delta=0$, with $\mathrm{I}_{\mathrm{p}}=$ 0.7-1.2 MA, $\mathrm{B}_{\mathrm{T}}=1.6-2 \mathrm{~T}$. Active divertor pumping was used in the majority of discharges, however the highest density and confinement discharge was also reproduced with passive wall pumping. Figure 1 shows the time traces from a discharge with the highest normalized density. With active pumping this discharge was highly reproducible. Density was increased with gas fueling at the rate of $\sim 14 \mathrm{~Pa} \mathrm{~m}^{3} / \mathrm{s}$ $(\sim 100$ Torr $\cdot 1 / \mathrm{s})$. Since nearly an equal amount of gas is exhausted at the divertor pump, the rate of density rise corresponds to only a few percent of the gas injection rate. A nearly similar discharge to that of Fig. 1 was obtained by reducing the gas puff rate by $50 \%$ with no active divertor pumping. The excess gas was removed, presumably, by divertor target plate or wall pumping.In all discharges, the outer leg of the divertor remained attached. In the highest density discharges the inner leg of the divertor was detached, with a low temperature $\left(\mathrm{T}_{\mathrm{e}} \sim 2 \mathrm{eV}\right)$ radiative zone outside the separatrix, near the inboard side of the X-point. In all high confinement cases the cold radiative zone was contained outside the separatrix.

During gas puffing, initially the pedestal density increases linearly with time. However, the pedestal density quickly saturates at $\sim 80 \%$ of the Greenwald limit while the line average density continues to rise up to $1.4 \times \mathrm{n}_{\mathrm{GW}}$. During the density rise, the stored thermal energy initially decreases, but eventually begins to increase linearly with time. The highest normalized densities were obtained at low q ( $\mathrm{q}_{95}=3$ ) and at moderately low auxiliary power, roughly twice the L-H transition threshold. In Section 4 we offer a possible explanation as to why operating at low $\mathrm{q}$ and low heating power is important for access to high normalized pedestal densities.

During the density ramp-up, the density profile temporarily becomes hollow. This can occur if the time constant for the pedestal density rise is shorter than the particle diffusion time scale across the plasma. Later in time the profile gradually becomes more peaked. In Fig. 2(a) density profiles during the fast pedestal density rise and later at a higher density are compared with the quasi steady state density profile of a low density discharge. The profiles are normalized to the same pedestal density. The density peaking factor, defined as the ratio of peak to line average density, reached $\leq 1.5$ at the end of the high density discharge, as compared to $\sim 1.3$ in the reference discharge. The additional density peaking at the highest densities is partly attributed to a lower particle diffusivity and a particle pinch in the center of the discharge [6]. In the vicinity of the separatrix, at high density, the density profile becomes very steep, as shown in Fig. 2(b). The half-width of the density profile in this region scales inversely with the pedestal density. This scaling is explained in Section 4.

All high confinement discharges displayed sawtooth activity and Type-I ELMs. In most cases tearing mode activity was also observed late in the discharge. The amplitudes of ELMs and sawtooth oscillations decreased with increasing density. The energy loss per ELM at high 
density, as shown in Fig. 3, was typically a factor of 5 lower in comparison with the expected value from a recent multidevice study at low density [5]. The reduction in energy loss per ELM is of great importance for design of the nextgeneration devices [7]. In addition to the above modes, which are normally detected in most $\mathrm{H}$-mode plasmas, during the fueling phase we also identified a new coherent mode which was radially localized to within $1 \mathrm{~cm}$ of the separatrix. The mode has a poloidal structure of $m \sim 40$, determined with beam emission spectroscopy. The frequency of the mode in the lab frame was of the order of $40 \mathrm{kHz}$. The origin of the mode has not been identified and will not be discussed further in this paper.

\section{Confinement and Stability Charac- teristics of High Density Plasmas}

A general characteristic of all high density discharges, as shown in Fig. 1, is that soon after the initiation of the gas fueling, confinement decreases. However, typically within one second of gas fueling, confinement begins to increase linearly with time. The rise in the stored energy is eventually arrested with the onset of a 3/2 MHD mode. The time behavior of stored energy appears as confinement dependence on density. However, a closer look reveals that the variation of stored energy is mainly due to the dynamics of the density profile and a stiff transport regime imposed by the ion temperature gradient (ITG) mode. With a stiff temperature profile, stored energy is expected to increase with the peaking of the density profile. In Fig. 4(a,b) we have plotted stored energy $\mathrm{W}$ and the normalized stored energy $\mathrm{W}_{\mathrm{N}}$, as a function of time, for several discharges. The normalized stored energy is defined as $\mathrm{W}_{\mathrm{N}} \equiv$ $\left(\mathrm{W} / 3 \overline{\mathrm{n}}_{\mathrm{e}} \mathrm{T}_{\mathrm{PED}} \mathrm{V}\right)$, where $\mathrm{T}_{\mathrm{PED}}$ is the pedestal electron temperature and $\mathrm{V}$ is the plasma volume. $\mathrm{W}_{\mathrm{N}}$ does not display the large scale excursions seen in the time behavior of $\mathrm{W}$, including the changes across the L- to $\mathrm{H}$-mode transition, suggesting that heat transport in these discharges is primarily due to the ITG turbulence. Using the gyro-Landau-fluid transport code GFL23 [8], we have simulated the stored energy of discharge of Fig. 1. For this exercise, the experimental density and heating profiles, as well as the pedestal parameters, all as a function of time were used as inputs to the code. The code then calculated the evolution of the temperature profiles which was then used to calculate the time evolution of the stored energy. Since the GLF23 code does not have a sawtooth model, the temperature profiles were forced to be flat within the $\mathrm{q}=1$ surface. The time evolution of the stored energy, 


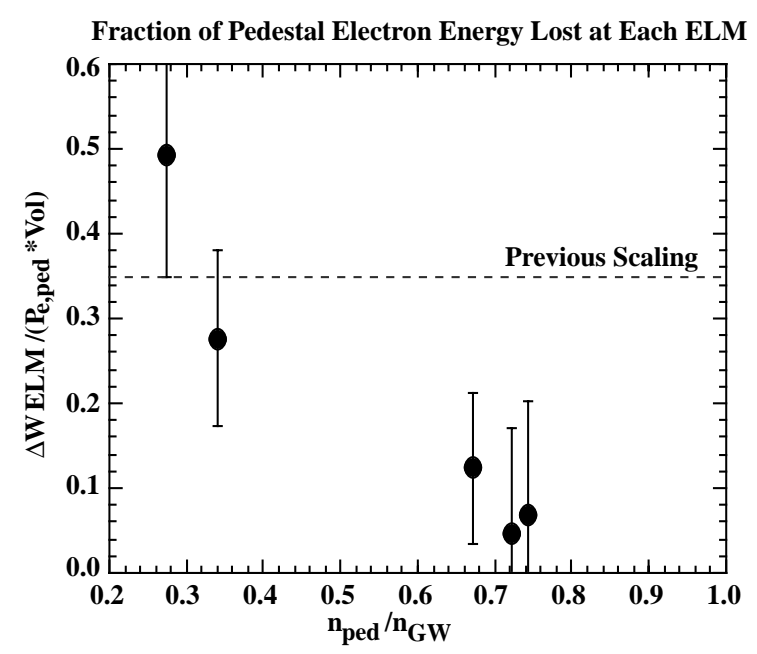

Fig. 3. The normalized ELM energy loss, $\Delta W_{n}$, as a function of normalized pedestal density. $\Delta W_{n}$ is given by the ELM energy divided by the pedestal electron pressure and the plasma volume.

calculated by the GLF23 code in Fig. 4(c), is in good agreement with the experimental measured profile.

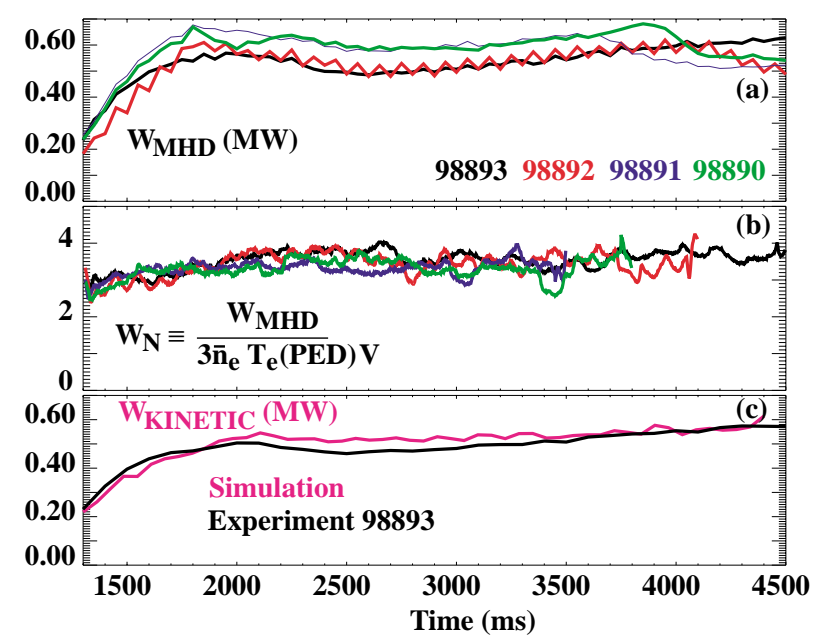

Fig. 4. (a) The plasma stored energy $W$ and (b) the normalized stored energy given by $W_{N} \equiv$ $W / 3 \overline{\mathrm{n}}_{e} T_{P E D} V .(b)$ The stored energy as predicted by the GLF23 code using the measured density profile and pedestal values as input. Note the good agreement with the measured plasma thermal energy.

In nearly all the high density cases, the density rise and improvement in confine-ment were ultimately terminated by the onset of a $3 / 2$ tearing mode (Fig. 1). Invariably, a cascade of higher modes, 6/5, 5/4, 4/3, as seen in Fig. 5(a) [6], preceded the 3/2 mode. In a sequence of discharges where the neutral heating power was varied shot by shot, the onset of the tearing mode occurred earlier in time with increasing power, and consequently at a lower density. However the onset of the mode was approximately at the same $\beta_{\mathrm{p}}$. In discharges where the heating power was sustained at a constant level, the mode ultimately saturated, with both density and stored energy reduced to a lower level. Both the classical and the neoclassical driving terms [9], as shown in Figs. 5(b,c) are increasing with time at the resonance surfaces. The magnitude and the sign of $\Delta^{\prime}$, calculated with the aid of PEST3 code [10], showed
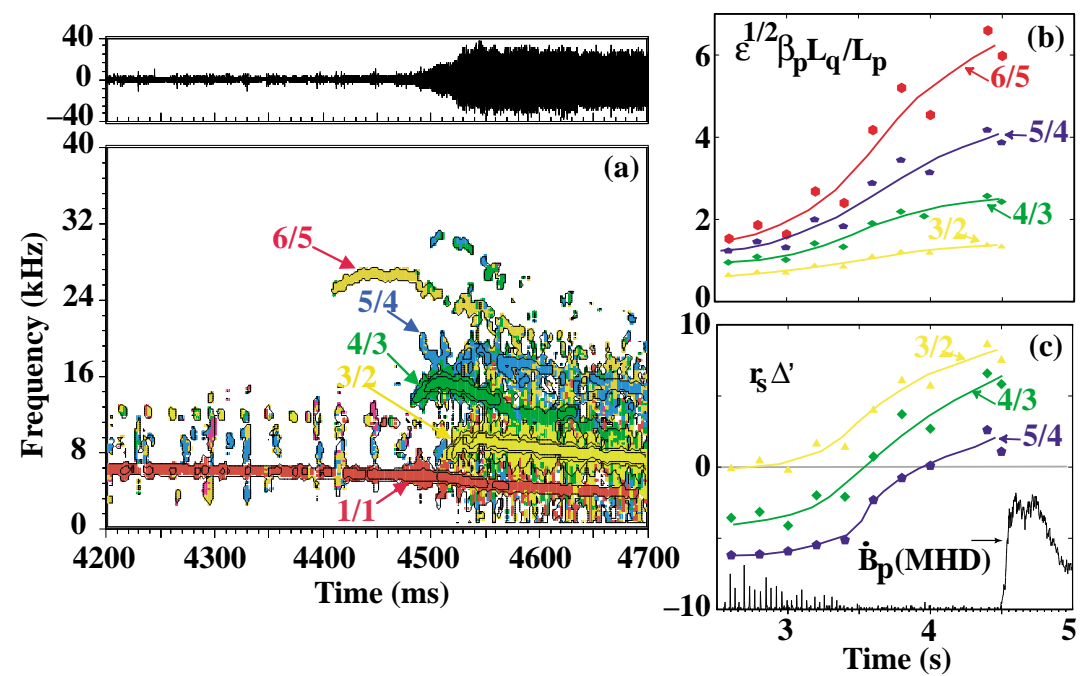

Fig. 5. (a) The spectrum of magnetic fluctuations as measured by Mirnov coils. A 6/5 mode first develops and eventually cascades down to a $3 / 2$ mode which saturates and ends the high density and high confinement phase. The drive terms for both the (b) neoclassical, $\varepsilon^{l / 2} \beta_{p} L_{q} / L_{p}$, and (c) classical, $r_{s} \Delta^{\prime}$, tearing modes increase with time. 
sensitivity to small changes in the input equilibria, however the increasing trend in $\Delta^{\prime}$ was reproducible. Thus, we are unable to state whether the modes observed are classical or neoclassical.

\section{Access to High Densities}

A key step in accessing high densities without confinement degradation is prevention of a cold radiative zone at the X-point [11-13]. The formation of a cold zone is correlated with divertor detachment [14] and the ensuing progression of a cold $\left(\mathrm{T}_{\mathrm{e}} \sim 2 \mathrm{eV}\right)$ ionization front towards the X-point. The cold zone forms at the same separatrix density at which the outer leg of the divertor detaches. In our earlier work, [11-13] densities up to $1.5 \mathrm{x} \mathrm{n}_{\mathrm{GW}}$ were obtained in ELM free H-mode plasmas (HITER89p 1.9) by maintaining the separatrix density well below detachment threshold with divertor pumping while fueling the plasma with pellets deposited well inside the separatrix. In absence of a core fueling source, the line average density can increase only if the separatrix density increases or the ratio of the pedestal to the separatrix density, $\eta=n_{P E D} / n_{\text {SEP }}$, increases. As we shall shortly demonstrate, divertor pumping can be used to increase $\eta$, at constant separatrix density. We will also offer reasons why with simultaneous puffing and pumping a higher separatrix density can be reached before the outer leg of the divertor detaches.

In the present experiments our approach to increasing $\eta$ was to fuel with gas away from the divertor and pump particles at the divertor. The fueling rate was selected such that the time constant for the density rise was longer than the density profile equilibration time. A key advantage of this arrangement is that the distribution of neutral source is shifted away from the X-point to areas with lower flux expansion. As a result, neutrals penetrate deeper inside the separatrix, causing $\eta$ to increase. In addition, a SOL flow, established by simultaneous gas puffing and pumping, should reduce the separatrix density relative to divertor plasma density, which would allow access to a higher separatrix density before reaching detachment.

To quantify these arguments, we have developed a simple model to calculate the density profile in the neighborhood of the separatrix. The model is presented in Appendix A. The model shows that $\eta$ can be written as a function of the dimensionless parameter $U=\left(\sigma \mathrm{V}_{\mathrm{e}} / \mathrm{V}_{\mathrm{H}}\right.$ $\left.\sqrt{\mathrm{D} \tau_{\|}}\right) \mathrm{n}_{\mathrm{PED}} \mathrm{f}\left(\theta_{0}\right)$ where, $\sigma, \mathrm{D}, \mathrm{V}_{\mathrm{e}}$ and $\mathrm{V}_{\mathrm{H}}$ are the separatrix values of ionization cross section, particle diffusivity, and electron and ion thermal speeds, respectively. $\tau_{\|}$is the average particle residence time in the portion of the SOL wrapping the closed flux surfaces. The quantity $f\left(\theta_{0}\right)$ represents the flux expansion at the location of a poloidally localized particle source. In our magnetic geometry $f\left(\theta_{0}\right)$ increases approximately by a factor of six from mid-plane to the $X$-point. For $U<1, \eta$ varies approximately as $2 / U$. Here for simplifying the discussion, we consider only the case where $\eta=2 / \mathrm{U}$. Then, $\eta=$ $2 /\left(\sigma \mathrm{V}_{\mathrm{e}} / \mathrm{V}_{\mathrm{H}} \sqrt{\mathrm{D}} \tau_{\|}\right) n_{\mathrm{PED}} \mathrm{f}\left(\theta_{0}\right)$. A prediction of this model is that the density scale length scales inversely with nPED, a result that is supported by our data shown in Fig. 2(b).

Now we are in a position to see how our experimental arrangement allows reaching a higher pedestal density for a fixed maximum separatrix density. Divertor pumping with gas puffing away from the divertor, such that the separatrix density is maintained, can reduce $f(\theta)$ and $\tau_{\|}$ simultaneously, thus increasing $\eta$. Under normal operating conditions, the neutral source is localized near the X-point [15], thus decreasing $f(\theta)$ by a factor of 6 . By moving the neutral source from $X$-point to midplane, $\eta$ can increase by more than a factor of $\sim 2.5$. The SOL flow established by the puff and pump also reduces $\tau_{\|}$.

The neutral source distribution depends on the details of the divertor wall and pumping geometry and the wall particle reflectivity. In a tightly baffled geometry with no effective particle removal mechanism at the target plate, fueling is more concentrated at the divertor, therefore the ratio of pedestal to separatrix density should decrease. Conversely, we expect to achieve higher line average densities by pumping the recycling sites near the private flux region. A significant redistribution of the neutral source occurs naturally at very high densities when the neutral leakage around the divertor plasma increases [16]. This effect becomes weaker with a tight baffle structure. 
The model of Appendix A also qualitatively explains two common experimental observations in H-mode plasmas: (1) fueling efficiency decreases rapidly with increasing density, and (2) occasionally additional fueling results in a drop in the line average density. The first of these is a consequence of the steepening of the density profile, which increases the particle loss rate. The second effect might be due to the ion temperature dependence of the parameter U. In typical DIII-D H-mode plasmas, ion temperature at the separatrix is normally much greater than the electron temperature [17]. At a sufficiently high separatrix density ions equilibrate with the electrons, and thus the mean-free path of charge exchange neutrals is reduced.

Finally there are two unrelated benefits of our experimental arrangement. With a strong SOL flow, induced by the simultaneous puff and pump, a significant fraction of the SOL heat flux is convected to the divertor, which tends to reduce the parallel temperature gradient, and thereby increases the separatrix density relative to the divertor density. In the extreme case of convection limited parallel heat transport, the divertor density approaches the upstream SOL density, whereas in the normal conduction limited regime divertor density is much greater than the upstream density and increases with the cube of the upstream density [18]. In our experiment we estimate the effect of convection is to increase the ratio of separatrix density to divertor density by roughly 10 percent. The second effect is due to reduced neutral density in the vicinity of the X-point, which helps to reduce the radiative cooling of the region.

\section{Discussion}

Two important parameters that define the density operating window in H-mode plasmas are power flow across the separatrix and toroidal field. In DIII-D, for a fixed plasma current, highest densities are achieved at low q and moderately low heating power, roughly twice the L-H power threshold. Figure 6 schematically shows the boundaries for attaining high normalized densities. The upper limit on power might be due to ELM particle loss. Since ELM frequency increases linearly with the heating power, $v_{\mathrm{ELM}} \sim \mathrm{P} / \mathrm{I}_{\mathrm{p}}^{1.5}[19]$, at high power ELM particle loss $\left[\sim n_{P E D} V_{E L M} \sim\left(n_{P E D} / I_{p}\right) x\right.$ P/I $\left.I_{p}^{0.5}\right]$ can exceed the transport losses. Near the $\mathrm{H}-\mathrm{L}$ transition power threshold, lowering power can actually increase the ELM losses. This is attributed to an increase in the duration of ELMs or short-lived L-mode phases that follow ELMs [12]. Since the H-L transition power threshold is proportional to the toroidal field, one gains by lowering the field proportionately to the power. The extent to which the toroidal field can be reduced is determined by the stability of tearing mode ( see the discussion in Section 3). Thus the operating window in the $\mathrm{B}_{\mathrm{T}}-\mathrm{P}_{\mathrm{inj}}$ plane, for a desired normalized density, as shown in Fig. 6 has a triangular shaped region.

Within the narrow operating window shown on Fig. 6, the achievable maximum density is determined by the plasma particle and neutral transport. Since particle diffusivity in the core scales inversely with $\mathrm{I}_{\mathrm{p}}[20]$, the core component of line average density (due to neutral beams or pellet sources) should increase linearly with $\mathrm{I}_{\mathrm{p}}$. On the other hand the pedestal density has weak dependence on D and no explicit dependence on $I_{p}$. Thus, in the absence of a core neutral particle source, the attainable normalized density should decrease with increasing $I_{p}$. Finally, up to

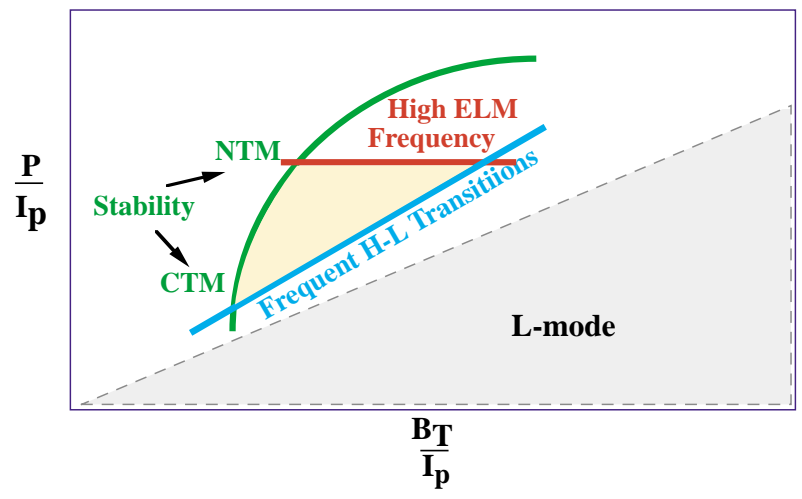

Fig. 6. A schematic diagram of the operational space for high density operation. this point we have ignored the possibility of a particle pinch. Our data strongly suggest the existence of a particle pinch near the plasma center [6]. Such pinch could significantly increase the attainable line average density. 


\section{Summary and Conclusions}

Densities up to $40 \%$ above the Greenwald limit were reproducibly obtained in gas fueled high confinement $\left(\mathrm{H}_{\text {ITER89p }}=2\right)$ ELMing H-mode DIII-D plasmas. These results show that there is no fundamental reason preventing high confinement $\mathrm{H}$-mode operation above the Greenwald limit. A combination of gas fueling away from the divertor and divertor pumping is used to access these densities. The heat transport in these discharges is accurately modeled by the GLF23 code, and similar to moderate density H-mode plasmas is characterized by a stiff temperature profile imposed by the ITG mode. A particle transport model was introduced that explains how divertor pumping helps access to higher densities. The main effects of simultaneous divertor pumping and gas fueling away from the divertor is to increases neutral penetration into the boundary plasma, resulting in a higher pedestal density. The increase in neutral penetration is due to : (1) a shift of the neutral particle source to regions of lower flux expansion, and (2) a lower particle confinement time in the SOL, which reduces the density gradient at the separatrix. We therefore conclude that further improvement in neutral penetration, by a more optimized fueling geometry or a high current low voltage neutral beam source, could result in even higher pedestal and line average densities. A highly desirable byproduct of operating at higher densities is that the amplitude of Type-I ELMs is a factor of 5 lower than the scaling result at lower densities predicts.

\section{Appendix A}

We have closely followed the approach used by Engelhart and Wagner et al. [21-23] which we will extend to a poloidally asymmetric magnetic configuration and neutral particle source.

In our model we neglect the neutral beam source in the region of interest, near the separatrix, since it is typically a factor of ten lower than the neutrals originating at wall. A few centimeters beyond the density pedestal, due to the short ionization mean free path of the edge neutrals, the neutral beam source becomes dominant. Thus the density profile has two characteristic scale lengths. The interior scale length is of the order of the minor radius, since the beam neutrals are normally deposited near the plasma center. The much shorter boundary plasmas density scale length is determined by the ionization profile of boundary neutrals.

Within the separatrix it is assumed that the plasma parameters are only functions of $\xi=1-\rho$, where $\rho$ is the normalized flux parameter, and that the plasma particle diffusion is determined by the electron density gradient in the real space. The neutral density, however is a function of both $\xi$ and the poloidal angle variable $\theta$. We also assume that in the region of interest, due to charge exchange collisions, neutrals travel with the local ion thermal speed and that the ratio of ion to electron speeds is a constant. The equation describing the neutral and plasma particles inside the separatrix are

$D d^{2} n_{e} / d \xi^{2}=\int n_{n} n_{e} \sigma V_{e} d \theta$

$\mathbf{V}_{\mathrm{H}}(\mathrm{dr} / \mathrm{d} \xi) \partial \mathrm{n}_{\mathrm{n}} / \partial \xi=-\mathrm{n}_{\mathrm{n}} \mathrm{n}_{\mathrm{e}} \sigma \mathrm{V}_{\mathrm{e}}$

where $\sigma$, and $\mathrm{D}$ are the ionization cross section and particle diffusivity, respectively, and are assumed constant and $\mathrm{dr} / \mathrm{d} \xi \equiv \mathrm{f}(\theta)$ is a function of the poloidal variable $\theta$. Integrating Eq. (2) over $\xi$ shows that the neutral distribution has the form $\mathrm{n}_{\mathrm{n}}=\mathrm{n}_{\mathrm{n}}(\theta, 0) \mathrm{g}(\xi)^{\mathrm{f}(\theta)}$. In order to simplify the algebra, we limit our discussion to the case that $n_{n}(\theta, 0)=\delta\left(\theta-\theta_{0}\right)$. After some algebra we arrive at the equation describing the electron density profile

$\mathrm{d}^{2} \mathrm{n}_{\mathrm{e}} / \mathrm{d} \xi^{2}=\mathrm{f}\left(\theta_{0}\right)\left(\sigma \mathrm{V}_{\mathrm{e}} / 2 \mathrm{~V}_{\mathrm{H}}\right)\left(\mathrm{dn}_{\mathrm{e}}^{2} / \mathrm{d} \xi\right)$

Except for the factor $\mathrm{f}\left(\theta_{0}\right)$, this result is the same as that given in Ref. [22]. 
$\exp \left(-\xi / \sqrt{\mathrm{D} \tau_{\|}}\right)[24]$, where $\tau_{\|}$is the average particle residence time in the portion of the SOL wrapping the separatrix. Using the boundary conditions of continuity of the density gradient across the separatrix and $\mathrm{n}_{\mathrm{e}}=\mathrm{n}_{\mathrm{PED}}$ for $\xi=-\infty$, we obtain the solution

$\mathrm{n}_{\mathrm{e}}=\mathrm{n}_{\mathrm{PED}} \mathrm{x} \tanh \left[\mathrm{C}-\left(\sigma \mathrm{V}_{\mathrm{e}} / 2 \mathrm{~V}_{\mathrm{H}}\right) \mathrm{f}\left(\theta_{0}\right) \mathrm{n}_{\mathrm{PED}} \xi\right]$,

$\eta=\mathrm{n}_{\mathrm{PED}} / \mathrm{n}_{\mathrm{SEP}}=1 / \tanh (\mathrm{C})$

where $C=0.5 \times \sinh ^{-1}(U)$ and $U=\left[\sigma V_{e} / V_{H}\left(\sqrt{D} \tau_{\|}\right)\right] f\left(\theta_{0}\right) n_{P E D}$.

Here we have interpreted the peak density as the pedestal density, since no other sources have been considered.

\section{Acknowledgment}

Work supported by the U.S. Department of Energy under Contract Nos. DE-AC0399ER54463, W-7405-ENG-48, DE-AC05-00OR22725, DE-AC04-94AL85000, and Grant Nos. DE-FG03-86ER53225, DE-FG02-92ER54139, and DE-FG03-95ER54294.

\section{References}

[1] HUGIL, S., Proc. 2nd Joint Varenna-Grenoble Symp., Brussels, Belgium (1980) p. 775.

[2] GREENWALD, M., TERRY, J.L., WOLFE, S.M., EJIMA, S., BELL, M.G., KAYE, S.M., NEILSON, G.H., Nucl. Fusion 28, 2199 (1988).

[3] THE JET TEAM, "Confinement Degradation of ELMy H-Modes at High Density and/or Radiated Power Fraction,” Fusion Energy 198 (Proc. 17th Int. Conf., Yokohama, 1998) IAEA, Vienna (1999) Vol. 1, p. 335.

[4] KAMADA, Y., et al., "Long Sustainment of JT-60U Plasmas With High Integrated Performance," ibid, p. 598.

[5] LEONARD, A.W., et al., J. Nucl. Mater. 266-269 (1999) 109.

[6] OSBORNE, T.H., et al., "Gas-Puff Fueled H-Mode Discharges With Good Energy Confinement Above the Greenwald Density Limit," to be published in J. Nucl. Mater.

[7] LEONARD, A.W., et al., "Tolerable ELMs at High Density in DIII-D," to be published in J. Nucl. Mater.

[8] WALTZ, R.E., et al., Phys. Plasmas 4 (1997) 2482.

[9] KOTSCHENREUTHER, M., HAZELTINE, R.D., MORRISON, P.J., Phys. Fluids 28 (1985) 294.

[10] PLETZER, A., BONDESON, A., DEWAR,R.L., J. Comput.Phys. 115, 530 (1994).

[11] MAHDAVI, M.A., et al., "Recent H-Mode Density Limit Experiments on DIII-D," Plasma Phys. Contr. Fusion 21 (1997) 1113.

[12] MAINGI, R., et al., Phys. Plasmas 4, 1752 (1997).

[13] MAHDAVI, M.A., et al., Proc. 16th Euro. Conf. on Controlled Fusion and Plasma Physics, Venice, Italy (1998) p. 355.

[14] PETRIE, T.W., Nucl. Fusion 37 (1977) 21.

[15] OWENS, L.W., "Origin and Spatial Distribution of Core Fueling in the DIII-D Tokamak," to be published in J. Nucl. Mater.

[16] FENSTERMACHER, M.E., et al., J. Nucl. Mater. 241-243 (1997) 666.

[17] PORTER, G.D., ISLER, R.C., BOEDO, J.A., ROGNLIEN, T.D., Phys. Plasmas 7 (2000) 3663.

[18] MAHDAVI, M., et al., Phys. Rev. Lett. 47 (1981) 1602.

[19] ZOHM, H., Plasma Phys. Contr. Fusion 38 (1996) 105.

[20] WADE, M.R., et al., Phys. Plasmas 2 (1995) 2357.

[21] ENGLEHARDT, W., FENEBERG, W., J. Nucl. Mater. 76-77 (1978) 518.

[22] WAGNER, F., LACKNER, K., "Phys. Plasmas - Wall Interactions in Controlled Fusion," Nato. ASI Series B, Physics Vol. 131, page 931

[23] STANGEBY, P., "The Plasma Boundary of Magnetic Fusion Devices," Inst. of Phys. Publishing, Bristol (2000) p. 175.

[24] YOKOMIZO, H., et al., Plasma Phys. and Contr. Nuclear Fusion Research 1982, Vol. III, IAEA, Vienna (1983) 173. 